\title{
A Study of Traditional Chinese Medicine Body Constitution Associated with Overweight, Obesity, and Underweight
}

\author{
Mihui Li, ${ }^{1,2}$ Shuming Mo,, ${ }^{2,3}$ Yubao Lv, ${ }^{1,2}$ Zihui Tang, ${ }^{1,2}$ and Jingcheng Dong \\ ${ }^{1}$ Department of Integrative Medicine, Huashan Hospital, Fudan University, Shanghai, China \\ ${ }^{2}$ The Institutes of Integrative Medicine, Fudan University, Shanghai, China \\ ${ }^{3}$ Department of Integrative Medicine, Huashan Hospital North, Fudan University, Shanghai, China
}

Correspondence should be addressed to Zihui Tang; dr_zhtang@yeah.net and Jingcheng Dong; dongjcfdu@yeah.net

Received 8 June 2017; Revised 21 August 2017; Accepted 11 September 2017; Published 19 October 2017

Academic Editor: Mariangela Rondanelli

Copyright (C) 2017 Mihui Li et al. This is an open access article distributed under the Creative Commons Attribution License, which permits unrestricted use, distribution, and reproduction in any medium, provided the original work is properly cited.

\begin{abstract}
Objective. The aim of the study was to investigate the associations among the nine types of body constitution in traditional Chinese medicine (TCM) with the outcomes of overweight, obesity, and underweight. Method. Participants aged 30 to 90 years were recruited from communities in Shanghai and assessed using a self-administered questionnaire pertaining to their demographics, lifestyles, and self-reported medical history. The data of 3748 participants with complete information was available for the analysis. Multinomial logistic regression (MLR) analysis was performed to determine the associations among the TCM constitution variables and the health outcomes. Results. The standards of classification and determination of the constitution in TCM were used to gauge the patients' constitution type. MLR revealed independent and significant associations among the Qi_Deficient and Yang_Deficient groups with the outcomes of overweight, obesity, and underweight $(P<0.10$ for all). MLR revealed independent and significant associations among the Qi_Deficient and Yang_Deficient groups with the outcomes of overweight, obesity, and underweight $(P<0.05$ for all). Conclusion. Our study revealed significant negative correlations between the Qi_Deficient and Yang_Deficient groups with the outcomes of overweight, obesity, and underweight. On the other hand, positive correlations were found between Phlegm_Dampness and the outcomes of overweight and obesity.
\end{abstract}

\section{Introduction}

Recently, the systematic constitutional theory of traditional Chinese medicine (TCM) was proposed, and improvements have been made to it gradually. In TCM, the body constitution is an individual character compatible with nature and the social environment; formed in the process of human growth and development, one's body constitution indicates one's susceptibility to diseases, metabolism, and response to stimuli. Under TCM, there are nine constitutions: Neutral, Qi_Deficient, Yang_Deficient, Yin_Deficient, Phlegm_Dampness, Blood_Stasis, Qi_Stagnation, and Special [1]. The standards pertaining to the classification and determination of the constitution in TCM were issued by the China Association for Traditional Chinese Medicine. Based on the symptoms presented, it is possible to evaluate one's constitution using the standard questionnaire [2].
The body mass index (BMI), which is derived from the mass and height of an individual, is an attempt to quantify the amount of tissue mass in an individual and accordingly categorize that person as being underweight, overweight, obese, or of normal weight. Obesity is a leading preventable cause of death worldwide [3] and is associated with various health issues, particularly cardiovascular diseases, type 2 diabetes mellitus (DM), certain types of cancer, and asthma $[3,4]$. The implications of the overweight health outcome are more controversial. Overweight can reduce life expectancy and increase the risk of oligospermia and azoospermia in men $[5,6]$. However, overweight was not associated with excess mortality [7]. Underweight is considered unhealthy and is an established risk factor for osteoporosis, even among young people [8].

Constitutional consideration not only determines the patient's susceptibility to certain pathogens and diseases but 
is also closely related to the development, treatment, and prognosis of diseases [9]. When an individual's constitution is evaluated, the congenital hereditary factor, physiological functions, psychological conditions, and social factor are usually taken into account, suggesting that the constitution has a close relationship with chronic diseases. Several studies have suggested that the constitution under TCM relates closely to chronic diseases, such as obesity, type $2 \mathrm{DM}$, hypertension (HTN), and metabolism syndrome (MetS) [1013]. However, few studies have systemically explored the relationship among the nine constitutions and overweight, obesity, and underweight. Based on previous studies, we assume that the constitutions may be associated with BMI. This study was aimed at investigating the relationship of body constitutions of TCM with overweight, obesity, and underweight in this sample.

\section{Methods}

As was mentioned earlier [14], we conducted a work to investigate the associations among chronic diseases and the nine body constitutions under TCM in a Chinese sample. We recruited more than 4,000 participants (30-90 years) from communities in Shanghai between 2011 and 2014. Participants with severe renal and hepatic function abnormalities and pregnant women were excluded. We obtained written consent from all participants before this study, which was approved by Ethical Committee of the Huashan Hospital and performed according to ethical standards in the Declaration of Helsinki. After excluding the incomplete responses, 3748 subjects with available data were used for the analysis.

All participants were completely evaluated for baseline characteristics. Similar to our previous studies [1416], the baseline data consisted of demographical information, lifestyle, and medical history. Hypertension (HTN), BMI, and DM were defined as in earlier studies [14-16]. According to Chinese criteria, BMI (weight $/$ height ${ }^{2}, \mathrm{~kg} / \mathrm{cm}^{2}$ ) was classified: underweight $\left(<18.0 \mathrm{~kg} / \mathrm{m}^{2}\right)$, normal weight $\left(\geq 18.0 \mathrm{~kg} / \mathrm{m}^{2}\right.$ and $\left.<24.0 \mathrm{~kg} / \mathrm{m}^{2}\right)$, overweight $\left(\geq 24.0 \mathrm{~kg} / \mathrm{m}^{2}\right.$ and $\left.<28.0 \mathrm{~kg} / \mathrm{m}^{2}\right)$, and obesity $\left(\geq 28.0 \mathrm{~kg} / \mathrm{m}^{2}\right)$ [17]. The TCM constitutions were assessed using a standard questionnaire recommended by the China Association for Traditional Chinese Medicine (Supplementary Document 1 in Supplementary Material available online at https://doi.org/10.1155/2017/ 7361896) [1].

2.1. Statistical Analysis. Differences analyses among participants categorized by BMI were performed using a one-way analysis of variance or $\chi^{2}$ test. Firstly, the association of the TCM constitution variables with the health outcomes was determined by using univariate regression analysis. Further, multinomial logistic regression (MLR) was employed to include a shared reference of normal weight in determining the associations among the constitutions with overweight, obesity, and underweight, control for potential confounding factors. The results were reported by using professional statistical software (Statistical Package for Social Sciences 16.0, SPSS, Chicago, IL, USA). A $P$ value of $<0.05$ was considered significant (two-sided tests). Odds ratios (OR) with $95 \%$ confidence intervals (CI) were calculated to assess the relative risk of body constitutions and BMI.

\section{Results}

The baseline characteristics of the 3748 participants are listed (Table 1). The mean age of the total sample was 69.33 years. The proportion of subjects with habits of drinking and smoking was $16.78 \%$ and $18.94 \%$, respectively. The prevalence of CAD, HTN, DM, and MetS was 11.18\%, 42.58\%, 16.84\%, and $10.80 \%$, respectively. The prevalence of overweight, obesity, and underweight in the total sample was $25.43 \%, 10.54 \%$, and $2.48 \%$, respectively. Significant differences were reported in weight, age, and drinking habits and the prevalence of HTN, DM, and MetS among the four groups categorized by BMI.

3.1. Univariate Analysis for BMI, Overweight, Obesity, and Underweight. Univariate analyses showed that the Qi_Deficient, Yang_Deficient, and Phlegm_Dampness variables were associated with BMI types significantly $(P$ value $<0.05$ for three, Table 2). The mean of BMI in the Neutral, Qi_Deficient, Yang_Deficient, and Phlegm_Dampness groups was $24.24 \mathrm{~kg} / \mathrm{m}^{2}, 23.66 \mathrm{~kg} / \mathrm{m}^{2}, 23.07 \mathrm{~kg} / \mathrm{m}^{2}$, and $25.10 \mathrm{~kg} / \mathrm{m}^{2}$ $(P<0.001$ for all, Figures $1(\mathrm{a})-1(\mathrm{c}))$, respectively. The proportions of normal BMI, overweight, and obesity were $37.52 \%, 34.07 \%$, and $31.38 \%$ in the Qi_Deficient group $(P=$ 0.044 and $P$ for trend $=0.043$, Figure 2(a)), respectively. The proportions were $34.49 \%, 23.80 \%$, and $19.20 \%$ in the Yang_Deficient group $(P<0.001$ and $P$ for trend $<0.001$, Figure $2(\mathrm{~b})$ ), respectively, and $10.62 \%, 15.54 \%$, and $19.78 \%$ in the Phlegm_Dampness group $(P<0.001$ and $P$ for trend $<0.001$, Figure $2(\mathrm{c}))$, respectively. Underweight participants accounted for $2.59 \%, 5.86 \%$, and $6.21 \%$ in the Neutral, Qi_Deficient, and Yang_Deficient groups $(P<0.001$ for all, Figures 3(a) and 3(b)), respectively. The univariate analysis revealed a negative correlation between the Qi_Deficient and Yang_Deficient groups and BMI, overweight, obesity, and underweight, whereas it indicated a positive correlation between Phlegm_Dampness and these outcomes.

3.2. Multiple Variable Analysis for BMI, Overweight, Obesity, and Underweight. The MLR detected significant associations of the Qi_Deficient, Yang_Deficient, and Phlegm_Dampness constitutions with the BMI outcomes, after controlling for potential confounding factors $(P<0.001$ for three, Table 3$)$. Moreover, the MLR with a shared reference of the normal weight BMI group reported that outcomes of overweight, obesity, and underweight were significant and independently associated with the Qi_Deficient and Yang_Deficient groups $(P<0.10$ for all, Table 4$)$, respectively. the results indicated that Phlegm_Dampness was associated with the outcomes of overweight and obesity $(P<0.01$ for two $)$ but was not associated with underweight $(P=0.948)$. No significant association was found in the other constitution groups (data not shown). 
TABLE 1: Baseline characteristics of participants.

\begin{tabular}{|c|c|c|c|c|c|c|}
\hline Variable & Normal BMI & Overweight & Obesity & Underweight & Total & $P$ value \\
\hline \multicolumn{7}{|c|}{ Demographical information } \\
\hline$N(\%)$ & $2307(61.55 \%)$ & $953(25.43 \%)$ & $395(10.54 \%)$ & $93(2.48 \%)$ & 3748 & - \\
\hline Age (years) & $69.37 \pm 7.57$ & $69.26 \pm 7.34$ & $68.71 \pm 7.27$ & $71.18 \pm 9.12$ & $69.33 \pm 7.53$ & 0.043 \\
\hline Gender (female) & $1260(54.61 \%)$ & $499(52.36 \%)$ & $224(56.71 \%)$ & $56(60.21 \%)$ & $2039(54.40 \%)$ & 0.156 \\
\hline Height (cm) & $161.28 \pm 8.06$ & $161.24 \pm 8.08$ & $160.34 \pm 8.86$ & $159.65 \pm 7.93$ & $161.26 \pm 15.19$ & 0.057 \\
\hline Weight (kg) & $58.17 \pm 7.68$ & $68.48 \pm 7.11$ & $77.4 \pm 9.01$ & $43.91 \pm 4.73$ & $62.37 \pm 10.82$ & $<0.001$ \\
\hline $\mathrm{SBP}(\mathrm{mmHg})$ & $134.57 \pm 22.62$ & $135.8 \pm 16.18$ & $131.86 \pm 16.55$ & $134.67 \pm 35.91$ & $134.44 \pm 19.98$ & 0.948 \\
\hline $\mathrm{DBP}(\mathrm{mmHg})$ & $64.5 \pm 23.26$ & $66.39 \pm 22.9$ & $78.64 \pm 30.46$ & $65 \pm 13.45$ & $67.12 \pm 24.07$ & 0.282 \\
\hline $\mathrm{HR}(\mathrm{bpm})$ & $74.09 \pm 14.21$ & $74.26 \pm 12.83$ & $73.74 \pm 12.23$ & $76.45 \pm 16.89$ & $74.19 \pm 13.73$ & 0.405 \\
\hline \multicolumn{7}{|l|}{ Lifestyle } \\
\hline Smoking (yes) & $424(18.38 \%)$ & $181(18.99 \%)$ & $90(22.78 \%)$ & $15(16.13 \%)$ & $710(18.94 \%)$ & 0.148 \\
\hline Alcohol (yes) & $368(15.95 \%)$ & $173(18.15 \%)$ & $82(20.76 \%)$ & $6(6.45 \%)$ & $629(16.78 \%)$ & 0.004 \\
\hline Dietary (unbalance) & $52(2.25 \%)$ & $21(2.20 \%)$ & $13(3.29 \%)$ & $4(4.30 \%)$ & $90(2.40 \%)$ & 0.127 \\
\hline Exercise (yes) & $1441(62.46 \%)$ & $577(60.55 \%)$ & $233(58.99 \%)$ & $50(53.76 \%)$ & $2301(61.39 \%)$ & 0.18 \\
\hline \multicolumn{7}{|l|}{ Medical history } \\
\hline HTN (yes) & $866(37.54 \%)$ & $485(50.89 \%)$ & $225(56.96 \%)$ & $20(21.51 \%)$ & $1596(42.58 \%)$ & $<0.001$ \\
\hline DM (yes) & $333(14.43 \%)$ & $192(20.15 \%)$ & $99(25.06 \%)$ & $7(7.53 \%)$ & $631(16.84 \%)$ & $<0.001$ \\
\hline CAD (yes) & $261(11.31 \%)$ & $104(10.91 \%)$ & $39(9.87 \%)$ & $15(16.13 \%)$ & 419 (11.18\%) & 0.343 \\
\hline MetS (yes) & $10(0.43 \%)$ & $277(29.06 \%)$ & $118(29.87 \%)$ & $0(0 \%)$ & $405(10.80 \%)$ & $<0.001$ \\
\hline
\end{tabular}

Note. SBP: systolic blood pressure; DBP: diastolic blood pressure; HR: heart rate; HTN: hypertension; DM: diabetes mellitus; CAD: coronary artery disease; and MetS: metabolic syndrome.

TABLE 2: Comparison of distribution of different body mass index among Neutral group and the other TCM constitution groups.

\begin{tabular}{|c|c|c|c|c|c|c|}
\hline Variable & Normal BMI & Overweight & Obesity & Underweight & Total & $P$ value \\
\hline Neutral & 1204 & 538 & 224 & 34 & 2010 & - \\
\hline Qi_Deficient & 723 & 278 & 103 & 49 & 1153 & $<0.001$ \\
\hline Yang_Deficient & 634 & 169 & 54 & 45 & 902 & $<0.001$ \\
\hline Yin_Deficient & 210 & 81 & 32 & 7 & 330 & 0.621 \\
\hline Phlegm_Dampness & 143 & 99 & 55 & 5 & 302 & $<0.001$ \\
\hline Damp_Heat & 76 & 30 & 17 & 1 & 124 & 0.385 \\
\hline Blood_Stasis & 44 & 20 & 9 & 1 & 74 & 0.992 \\
\hline Qi_Stagnation & 31 & 13 & 8 & 1 & 53 & 0.832 \\
\hline Special & 49 & 25 & 8 & 3 & 85 & 0.511 \\
\hline
\end{tabular}

Note. TCM: traditional Chinese medicine.

\section{Discussion}

The associations of the TCM constitutions with the overweight, obesity, and underweight BMI outcomes were analyzed for a sample of individuals from the Chinese population. Several studies on the body constitution in TCM have been conducted in recent years, and most of the study participants were from the central or western region of China [18-20]. Obesity has become a major global health challenge due to its increasing prevalence and associated health risks $[21,22]$. Our data showed that $10.13 \%$ of adults suffered from obesity, similar to the figure reported for our sample. Much of the research has demonstrated that unhealthy lifestyles in terms of habits such as alcohol consumption lead to overweight and obesity, which in turn are closely related to many chronic diseases [23-25]. We used a standard questionnaire to evaluate the body constitution as per TCM in a large sample of Chinese from rural and urban communities of Shanghai. Moreover, to the best of our knowledge, this is the first study to employ the MLR approach of including a shared reference of normal weight to investigate the associations among the TCM body constitution types and the outcomes of overweight, obesity, and underweight. It is critical for integrative medicine practitioners to understand the clinical significance of TCM for these outcomes. This is partly because prevention (known in TCM as "Zhi Wei Bing") is the one of the most important essences of TCM, indicating the preventive treatment of diseases. The body constitution in TCM could indicate the patients' overall condition and could form the basis of prevention (i.e., Zhi Wei Bing) theory; thus, it has attracted much attention from researchers and clinicians. 


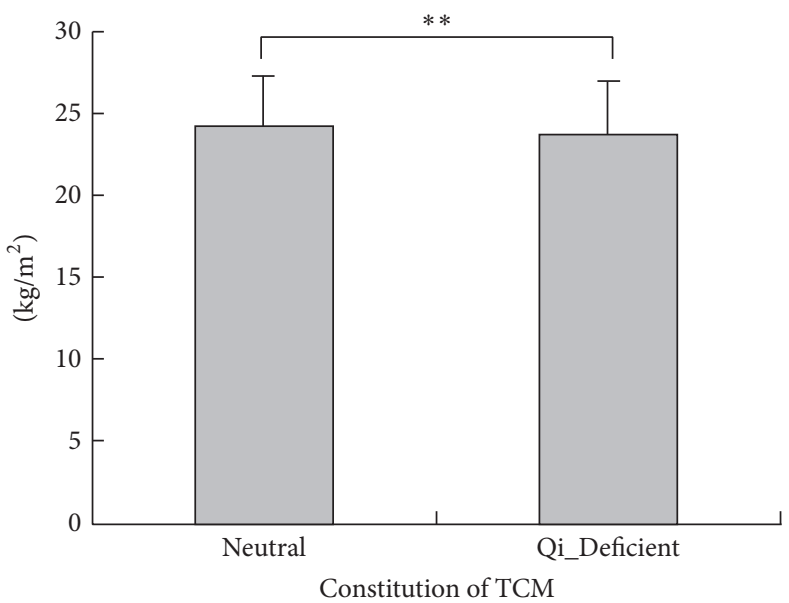

(a)

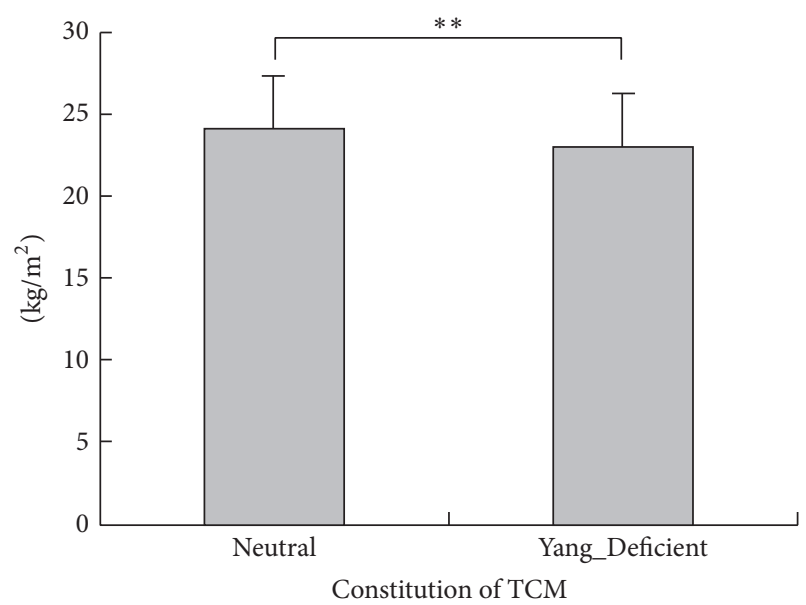

(b)

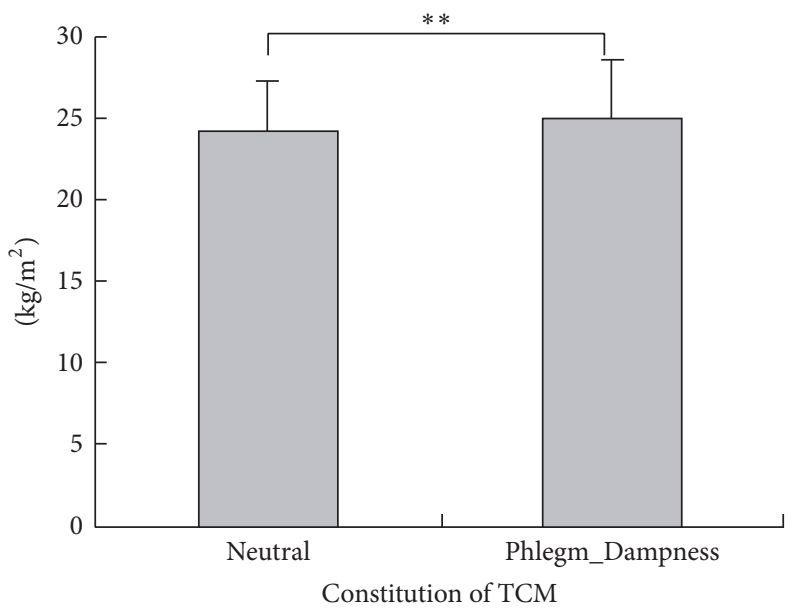

(c)

FIGURE 1: Comparison of body mass index among Neutral group and the other traditional Chinese medicine constitution groups. (a) The mean of body mass index was $24.24 \mathrm{~kg} / \mathrm{m}^{2}$ and $23.66 \mathrm{~kg} / \mathrm{m}^{2}$ in Neutral group and Qi_Deficient group $(P<0.001)$, respectively; (b) the mean of body mass index was $24.24 \mathrm{~kg} / \mathrm{m}^{2}$ and $23.07 \mathrm{~kg} / \mathrm{m}^{2}$ in Neutral group and Yang_Deficient group $(P<0.001)$, respectively; (c) the mean of body mass index was $24.24 \mathrm{~kg} / \mathrm{m}^{2}$ and $25.10 \mathrm{~kg} / \mathrm{m}^{2}$ in Neutral group and Phlegm_Dampness group $(P<0.001)$, respectively. ${ }^{* *}$ Very significant.

TABLE 3: Multinomial logistic analysis to include TCM constitution for BMI.

\begin{tabular}{lccccc}
\hline Variable & Beta & S.E & $t$ value & $P$ value & $95 \%$ CI for Beta \\
\hline Qi_Deficient & -0.578 & -0.087 & -4.85 & $<0.001$ & $-0.812--0.345$ \\
Yang_Deficient & -1.112 & -0.162 & -8.794 & $<0.001$ & $-1.36--0.864$ \\
Phlegm_Dampness & 0.85 & 0.089 & 4.348 & $<0.001$ & $0.466-1.233$ \\
\hline
\end{tabular}

Note. Multiple variable analysis adjusted for age, gender, exercise, dietary, smoking, alcohol, HTN, DM, and CAD; TCM: traditional Chinese medicine and BMI: body mass index.

Our research yielded interesting findings. Overweight, obesity, and underweight outcomes were significant and independent and were negatively correlated with the Qi_deficient and Yang_Deficient groups. The univariate analysis indicated that the BMI outcomes were lower in the Qi_Deficient and Yang_Deficient groups as compared to the Neutral group $(P<0.05$ for all). Moreover, multivariate analysis, controlling for potential confounders, reported that these two constitution types were significantly and independently associated with BMI and its outcomes $(P<0.010$ for all $)$. The most distinguished feature of the Yang_Deficiency type of constitution is that people of this type usually feel cold. People who are overweight and suffering from obesity have excessive subcutaneous fat, the function of which is to maintain body temperature and help them resist colder temperatures. Yanbo et al. made a similar conclusion, finding that a slim body was significantly positively correlated to the Yang_Deficiency type of constitution [26]. The study indicated that 2 genes were 

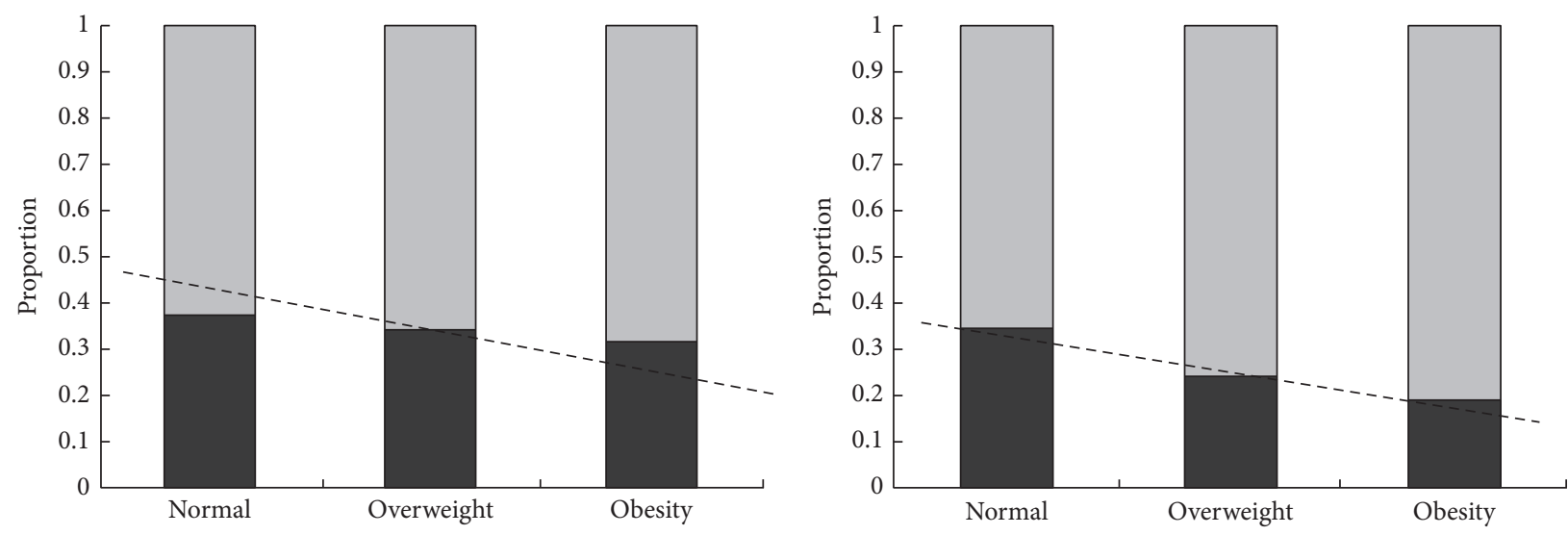

Qi_Deficient

Neutral

(a)

(b)

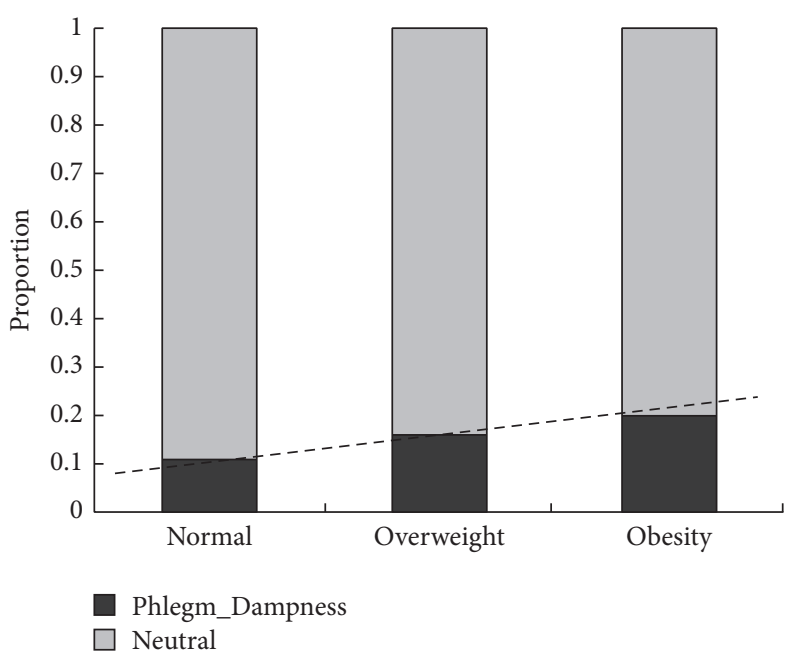

(c)

Figure 2: Distribution of normal BMI, overweight, and obesity among Neutral group and the other TCM constitution groups. (a) The proportion of normal BMI, overweight, and obesity was 37.52\%, 34.07\%, and 31.38\% in Qi_Deficient group $(P=0.044$ and $P$ for trend $=$ 0.043), respectively; (b) the proportion of normal BMI, overweight, and obesity was 34.49\%, 23.80\%, and 19.20\% in Yang_Deficient group $(P<0.001$ and $P$ for trend $<0.001)$, respectively; and (c) the proportion of normal BMI, overweight, and obesity was $10.62 \%, 15.54 \%$, and $19.78 \%$ in Phlegm_Dampness group $(P<0.001$ and $P$ for trend $<0.001)$, respectively.

TABLE 4: Multinomial logistic regression analysis to include TCM constitution for overweight, obesity, and underweight.

\begin{tabular}{lcccccc}
\hline TCM constitution & Outcome & Beta & S.E & $P$ value & OR & 95\% CI \\
\hline \multirow{3}{*}{ Qi_Deficient } & Overweight & -0.149 & 0.085 & 0.098 & 0.862 & $0.785-1.018$ \\
& Obesity & -0.312 & 0.133 & 0.019 & 0.732 & $0.564-0.951$ \\
& Underweight & 0.784 & 0.243 & 0.001 & 2.191 & $1.361-3.529$ \\
\hline \multirow{3}{*}{ Yang_Deficient } & Overweight & -0.509 & 0.104 & $<0.001$ & 0.601 & $0.49-0.737$ \\
& Obesity & -0.781 & 0.165 & $<0.001$ & 0.458 & $0.332-0.633$ \\
& Underweight & 0.812 & 0.246 & 0.001 & 2.253 & $1.391-3.65$ \\
\hline \multirow{3}{*}{ Phlegm_Dampness } & Overweight & 0.448 & 0.142 & 0.002 & 1.565 & $1.184-2.068$ \\
& Obesity & 0.745 & 0.179 & $<0.001$ & 2.107 & $1.485-2.991$ \\
& Underweight & -0.035 & 0.542 & 0.948 & 0.965 & $0.334-2.793$ \\
\hline
\end{tabular}

Note. Multiple variable analysis adjusted for age, gender, exercise, dietary, smoking, alcohol, HTN, DM, and CAD; TCM: traditional Chinese medicine. 


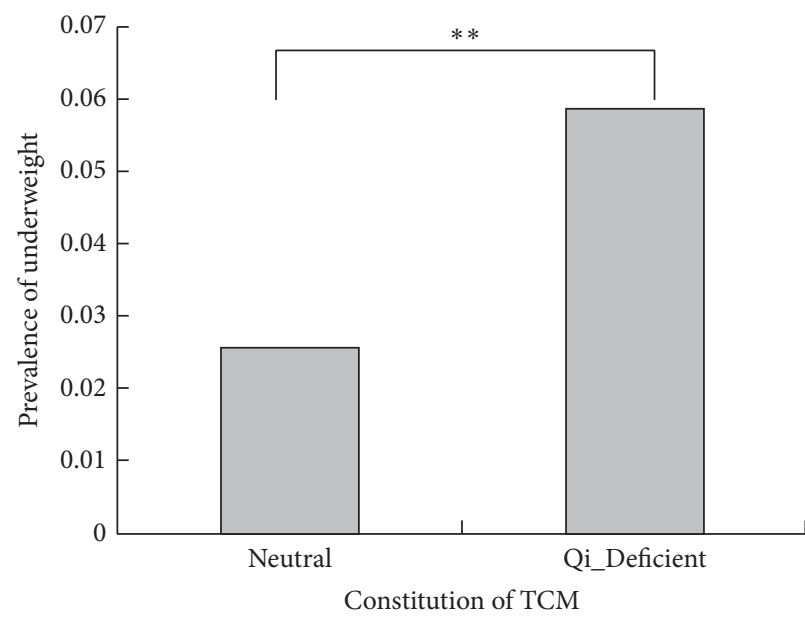

(a)

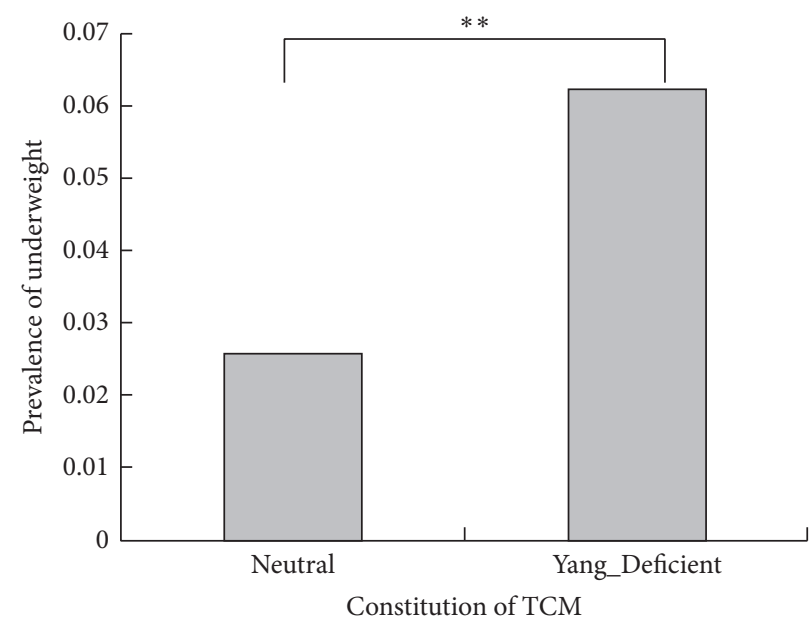

(b)

FIGURE 3: Comparison of prevalence of underweight among Neutral group and the other traditional Chinese medicine constitution groups. (a) The prevalence of underweight was $2.59 \%$ and $5.86 \%$ in Neutral group and Qi_Deficient group $(P<0.001)$, respectively; (b) the prevalence of underweight was $2.59 \%$ and $6.21 \%$ in Neutral group and Yang_Deficient group $(P<0.001)$, respectively. ${ }^{* *}$ Very significant.

upregulated and 38 genes were downregulated significantly among individuals with the Qi_Deficiency type of constitution as compared with those having a Neutral constitution; further, the downregulated genes including the ATP binding gene, GTP binding gene, MHC protein binding gene, and AMP kinase were involved in ion transport, cholesterol synthesis, and fatty acid synthesis [27]. The downregulation of these genes might imply reduced heat production, a lack of appetite, and weight loss. However, there are also some contradictory research results showing a positive relationship between Qi_Deficiency and obesity [18, 28]. In view of the complexity of the body constitution in TCM, we consider the proportion of unbalanced constitution types in one person at the same time; accordingly, the Qi_Deficiency constitution could coexist with other constitution types in one person, thus leading to different results [29].

Another interesting finding was that the Phlegm_Dampness type of constitution was positively, significantly, and independently correlated with overweight or obesity, but it was not associated with underweight. Overweight people are phlegmatic according to the classical theories of TCM. Our results showed that the Phlegm_Dampness constitution is positively associated with obesity and overweight, which is consistent with the findings of previous research [30,31]. The Phlegm_Dampness constitution type is very common in the study population. Several studies researched the differences between the Phlegm_Dampness and non-Phlegm_Dampness types from genome and single nucleotide polymorphisms (SNP), indicating that several genes and SNPs are significantly different between the Phlegm_Dampness and Neutral constitutions [32-34]. The identified genes are involved in enzyme activities and sterol transporter activities as well as in the process of lipid metabolism, cholesterol metabolism, brown fat cell differentiation, gluconeogenesis, and thermoregulation, indicating that an individual with
Phlegm_Dampness constitution is susceptible to metabolic disorders including obesity [35]. Subjects with the Phlegm_Dampness constitution type usually manifest a series of comprehensive characteristics such as obesity, a preference for greasy food, large bellies, fatigue, and a slippery pulse [32]. Their constitution is affected by an initial endowment as well as environmental and lifestyle factors. Qi et al. found that greasy food, lack of exercise, irregular sleep, and smoking were all important determinants of the Phlegm_Dampness constitution [30]. Research in genomics has shown four upregulated genes and six downregulated genes in the Phlegm_Dampness constitution [35]. Qi et al. found the discrepancy between the Phlegm_Dampness constitution and non-Phlegm_Dampness constitution of obesity in ATP binding gene including Janus kinase 2, nuclear receptor protein, and $\mathrm{CDC1}$ [36], providing molecular biological evidence for the association between the Phlegm_Dampness constitution and overweight and obesity. In clinical practice, we could apply the constitution of the Chinese medicine scale to estimate the risks of the Phlegm_Dampness constitution in terms of obesity and overweight and its related diseases. The constitutional theory of TCM recognizes individual differences from a synthetic and dynamic perspective based on morphological, functional, and psychological characteristics. Further research on the Phlegm_Dampness constitution is critical to enable the prediction and prevention of overweight, obesity, or related diseases.

This study has some limitations. On the one hand, the research sample was not recruited via a rigorous random sampling method, which means that the results are not sufficiently representative of the population in Shanghai. On the other hand, the factors affecting the constitution were not comprehensive, such as education, and psychological factors should be taken into account in future research. 


\section{Conclusion}

Our findings provided evidence that the Qi_Deficient and Yang_Deficient constitution types were significantly and independently associated with the outcomes of overweight, obesity, and underweight. Phlegm_Dampness was positively, significantly, and independently correlated with the outcomes of overweight and obesity. The Qi_Deficient or Yang_Deficient participants were less frequently found to be overweight or obese as compared with participants with Neutral constitution. A higher prevalence of overweight or obesity was found in Phlegm_Dampness participants. These findings may provide insights into clinical practice toward the prevention and diagnosis of the overweight, obesity, and underweight health outcomes.

$\begin{array}{ll}\text { Abbreviations } \\ \text { TCMC: } & \text { Traditional Chinese medicine constitution } \\ \text { SBP: } & \text { Systolic blood pressure } \\ \text { DM: } & \text { Diabetes mellitus } \\ \text { CAD: } & \text { Coronary artery disease } \\ \text { MetS: } & \text { Metabolic syndrome } \\ \text { HTN: } & \text { Hypertension } \\ \text { BMI: } & \text { Body mass index } \\ \text { DM: } & \text { Diabetes mellitus } \\ \text { OGTT: } & \text { Oral glucose tolerance test } \\ \text { MR: } & \text { Multivariable regression } \\ \text { OR: } & \text { Odds ratios } \\ \text { CI: } & \text { Confidence intervals. }\end{array}$

\section{Data Access}

The datasets generated and/or analysed during the current study are not publicly available due to private information but are available from the corresponding author on reasonable request. Datasets are from the study whose authors may be contacted at Center of Bioinformatics and Biostatistics, Institutes of Integrative Medicine, Fudan University.

\section{Ethical Approval}

All procedures performed in studies involving human participants were in accordance with the ethical standards of the institutional and/or national research committee and with the 1964 Helsinki declaration and its later amendments or comparable ethical standards. This study was approved by the Ethics Committee of Huashan Hospital, Shanghai, China. The methods were carried out in accordance with the approved guidelines.

\section{Consent}

Written informed consent was obtained from all patients before the study.

\section{Conflicts of Interest}

The authors declared that there are no conflicts of interest.

\section{Authors' Contributions}

Mihui Li and Yubao Lv drafted the manuscript. Shuming Mo and Zihui Tang participated in the design of the study and performed the statistical analysis. Zihui Tang and Jingcheng Dong conceived the study, participated in its design and coordination, and helped to draft the manuscript. All authors read and approved the final manuscript. Mihui $\mathrm{Li}$ and Shuming Mo have contributed equally to this work.

\section{Acknowledgments}

The authors acknowledge grants from the Clinical Medicine Foundation of Huashan Hospital; grants from the Institutes of Integrative Medicine of Fudan University to support the study; Development Project of Shanghai Peak DisciplinesIntegrative Medicine (20150407), Shanghai; and China Postdoctoral Science Foundation Funded Project (2017M611461).

\section{References}

[1] J. Wang, Y. Li, C. Ni, H. Zhang, L. Li, and Q. Wang, "Cognition research and constitutional classification in Chinese medicine," The American Journal of Chinese Medicine, vol. 39, no. 4, pp. 651660, 2011.

[2] Medicine CaoTC, "Classification and Determination of Constitution in TCM(ZYYXH/T157-2009)," World Journal of Integrated Traditional and Western Medicine, vol. 4, pp. 303-304, 2009.

[3] D. W. Haslam and W. P. T. James, “Obesity," The Lancet, vol. 366, no. 9492, pp. 1197-1209, 2005.

[4] M. Poulain, M. Doucet, G. C. Major et al., "The effect of obesity on chronic respiratory diseases: pathophysiology and therapeutic strategies," Canadian Medical Association Journal, vol. 174, no. 9, pp. 1293-1299, 2006.

[5] A. Peeters, J. J. Barendregt, F. Willekens et al., "Obesity in adulthood and its consequences for life expectancy: a life-table analysis," Annals of Internal Medicine, vol. 138, no. 1, pp. 24-32, 2003.

[6] N. Sermondade, C. Faure, L. Fezeu et al., "BMI in relation to sperm count: An updated systematic review and collaborative meta-analysis," Human Reproduction Update, vol. 19, no. 3, Article ID dms050, pp. 221-231, 2013.

[7] K. M. Flegal, B. I. Graubard, D. F. Williamson, and M. H. Gail, "Excess deaths associated with underweight, overweight, and obesity," Journal of the American Medical Association, vol. 293, no. 15, pp. 1861-1867, 2005.

[8] C. G. Gjesdal, J. I. Halse, G. E. Eide, J. G. Brun, and G. S. Tell, "Impact of lean mass and fat mass on bone mineral density: the Hordaland Health Study," Maturitas, vol. 59, no. 2, pp. 191-200, 2008.

[9] Y. Sun, P. Liu, Y. Zhao et al., "Characteristics of TCM constitutions of adult Chinese women in Hong Kong and identification of related influencing factors: A cross-sectional survey," Journal of Translational Medicine, vol. 12, no. 1, article no. 140, 2014.

[10] R. Lin, C. Tzeng, Y. Lee et al., "Acute effect of electroacupuncture at the Zusanli acupoints on decreasing insulin resistance as shown by lowering plasma free fatty acid levels in steroidbackground male rats," BMC Complementary and Alternative Medicine, vol. 9, article 26, 2009. 
[11] S. J. Grant, D. H.-T. Chang, J. Liu, V. Wong, H. Kiat, and A. Bensoussan, "Chinese herbal medicine for impaired glucose tolerance: a randomized placebo controlled trial," BMC Complementary and Alternative Medicine, vol. 13, article 104, 2013.

[12] A. Hussain, M. K. Yadav, S. Bose et al., "Daesiho-tang is an effective herbal formulation in attenuation of obesity in mice through alteration of gene expression and modulation of intestinal microbiota," PLoS ONE, vol. 11, no. 11, Article ID e0165483, 2016.

[13] Y. Zheng, J. Zhang, Y. Wang et al., "Acupuncture Decreases Blood Pressure Related to Hypothalamus Functional Connectivity with Frontal Lobe, Cerebellum, and Insula: A Study of Instantaneous and Short-Term Acupuncture Treatment in Essential Hypertension," Evidence-based Complementary and Alternative Medicine, vol. 2016, Article ID 6908710, 2016.

[14] J. Liu, F. Xu, N. Mohammadtursun, Y. Lv, Z. Tang, and J. Dong, "The Analysis of Constitutions of Traditional Chinese Medicine in Relation to Cerebral Infarction in a Chinese Sample," The Journal of Alternative and Complementary Medicine, 2017.

[15] C.-X. Yu, X.-Z. Zhang, K. Zhang, and Z. Tang, "A cross-sectional study for estimation of associations between education level and osteoporosis in a Chinese men sample Epidemiology of musculoskeletal disorders," BMC Musculoskeletal Disorders, vol. 16, no. 1, article no. 382, 2015.

[16] G.-Z. Qing, Y. Lu, T. Yi, K.-Q. Zhang, and Z.-H. Tang, “The relationship of frequency of meat consumption and osteoporosis in Chinese postmenopausal women," International Journal of Clinical and Experimental Medicine, vol. 8, no. 11, pp. 2113021137, 2015.

[17] B.-F. Zhou, "Predictive values of body mass index and waist circumference for risk factors of certain related diseases in Chinese adults-study on optimal cut-off points of body mass index and waist circumference in Chinese adults," Biomedical and Environmental Sciences, vol. 15, no. 1, pp. 83-96, 2002.

[18] H. Ping, Z. Qing, and S. Hongtao, "Research on Constitutions Types and Features of theObese People," Western Journal of Traditional Chinese Medicine, vol. 29, pp. 63-66, 2016.

[19] S.-L. Yao, Z.-Z. Zhang, X.-S. Yang et al., "Analysis of composite traditional Chinese medicine constitution: An investigation of 974 volunteers," Journal of Chinese Integrative Medicine, vol. 10, no. 5, pp. 508-515, 2012.

[20] Y. L. Feng, G. Y. Zheng, and C. Q. Ling, "The investigation of the correlation between metabolic syndrome and chinese medicine constitution types in senior retired military personnel of the people's liberation army," Chinese journal of integrative medicine, vol. 18, no. 7, pp. 485-489, 2012.

[21] M. Ng, T. Fleming, and M. Robinson, "Global, regional, and national prevalence of overweight and obesity in children and adults during 1980-2013: a systematic analysis for the Global Burden of Disease Study 2013," The Lancet, vol. 384, no. 9945, pp. 766-781, 2014.

[22] Z. Wenhua, Z. Yi, H. Jianping et al., "Economic burden of obesity related chronic disease in China," Chi J Epidemiol, vol. 27, pp. 555-559, 2006.

[23] X. Hanpeng, W. Xintong, C. Naichun et al., "The relationship between lifestyle and obesity," Acta Academiae, vol. 45, pp. 508510, 2009.

[24] D. Vancampfort, M. Hallgren, J. Mugisha et al., “The prevalence of metabolic syndrome in alcohol use disorders: A systematic review and meta-analysis," Alcohol and Alcoholism, vol. 51, no. 5, Article ID agw040, pp. 515-521, 2016.
[25] F. Montazerifar, A. Bolouri, R. S. Paghalea, M. K. Mahani, and M. Karajibani, "Obesity, serum resistin and leptin levels linked to coronary artery disease," Arquivos Brasileiros de Cardiologia, vol. 107, no. 4, pp. 348-353, 2016.

[26] Z. Yanbo, W. Qi, and Y. Shilin, "Study on the related influencing factors of Yang-deficiency constitution in Chinese medicine," Journal of Traditional Chinese Medicine, vol. 48, pp. 1113-1124, 2007.

[27] W. Ji, L. Yingshuai, L. Liru et al., "Genomics on nine types of TCM costituion," China Journal of Traditional Chinese Medicine, vol. 29, pp. 3871-3873, 2014.

[28] Z. Yanbo, W. Qi, W. Chengyu et al., "Logistic regression analysis on relationship between traditional Chinese medicine constitutional types and overweight or obesity," Journal of Chinese Integrative Medicine, vol. 8, pp. 1023-1028, 2010.

[29] Z. Yanbo, "Constitutional classification and comprehensive evaluation in Chinese medicine," China Journal of Traditional Chinese Medicine and Pharmacy, vol. 27, pp. 40-42, 2012.

[30] W. Qi, Z. Yangbo, O. Hideki et al., "Study on related influencing factors of phlegm_wetness constitution in Chinese medicine," Journal of Beijing University of Traditional Chinese Medicine, vol. 31, pp. 10-13, 2008.

[31] W. Qi, Y. Jianong, Z. Yangbo et al., "Research on diagnosis standard of phlegm-dampness constitution of TCM," China Journal of Traditional Chinese Medicine, vol. 21, pp. 73-75, 2006.

[32] W. Dongpo and W. Qi, "Analysis and expectation of the current research on phlegm-dampness constituion," China Journal of Traditional Chinese Medicine and Pharmacy, vol. 23, pp. 5-8, 2008.

[33] G. Haiyang, G. Jinghong, and Q. Wang, "Peripheral blood gene expression profile of Chinese adult obesities by gene chip technique," Journal of Clinical Rehabilitative Tissue Engineering Research, vol. 12, pp. 4797-4800, 2008.

[34] Y. Wu, Y. Cun, J. Dong et al., "Polymorphisms in PPARD, PPARG and APM1 associated with four types of traditional chinese medicine constitutions," Journal of Genetics and Genomics, vol. 37, no. 6, pp. 371-379, 2010.

[35] J. Wang, Q. Wang, L. Li et al., "Phlegm-dampness constitution: genomics, susceptibility, adjustment and treatment with traditional Chinese medicine," The American Journal of Chinese Medicine, vol. 41, no. 2, pp. 253-262, 2013.

[36] W. Qi, G. Haiyang, and G. Jinghong, "Study on characteristics of peripheral blood gene expression profile in the obesity with phlegm-dampness constitution," Journal of Traditional Chinese Medicine, vol. 47, pp. 851-858, 2006. 


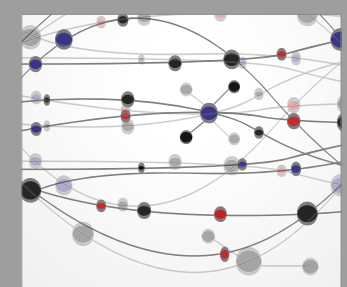

The Scientific World Journal
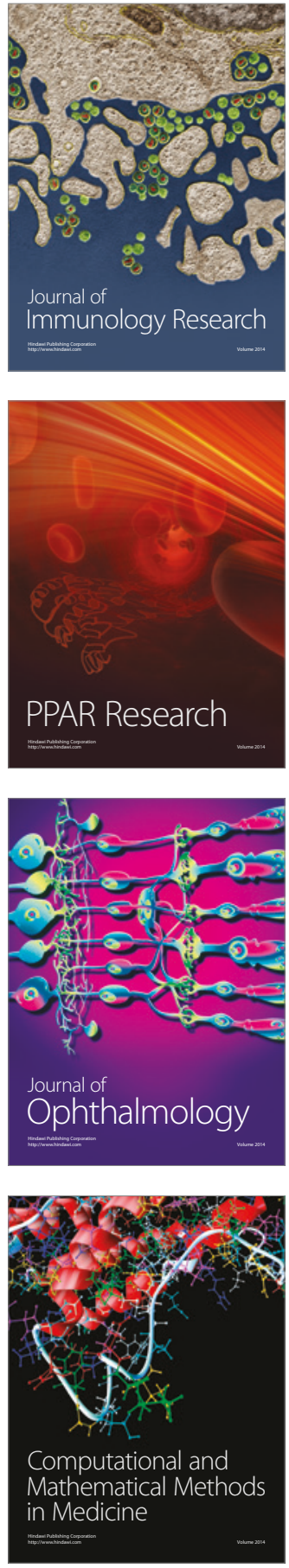

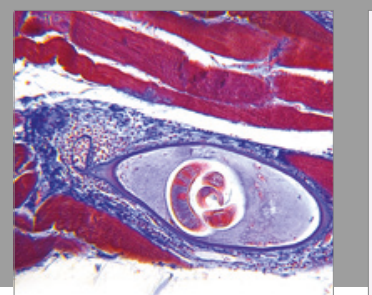

Gastroenterology Research and Practice
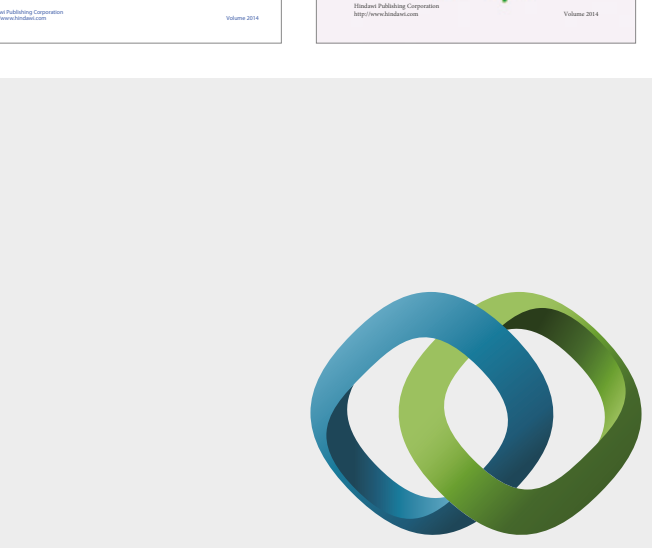

\section{Hindawi}

Submit your manuscripts at

https://www.hindawi.com
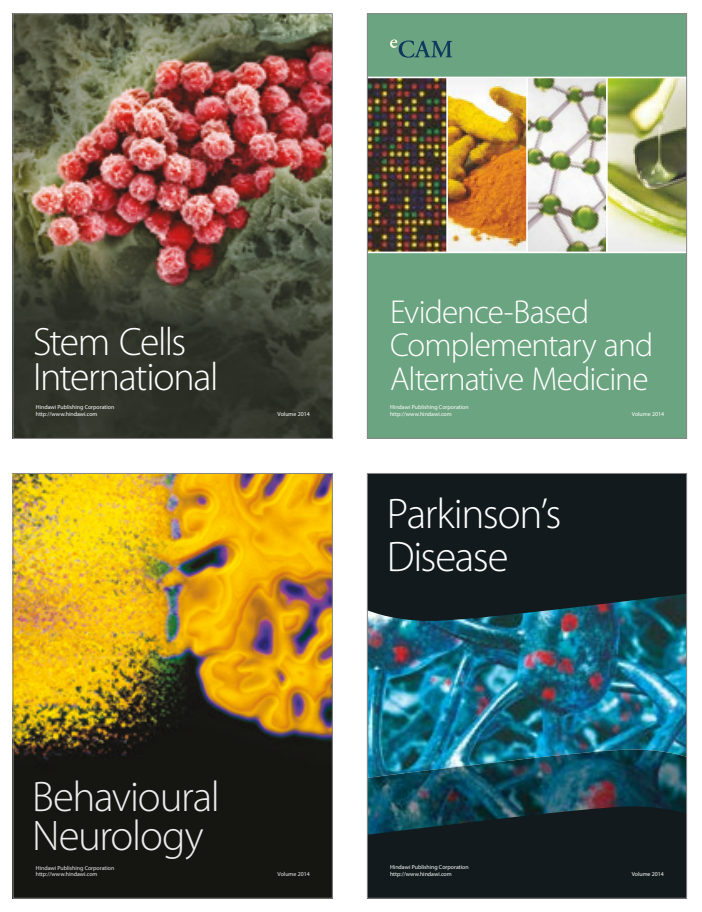
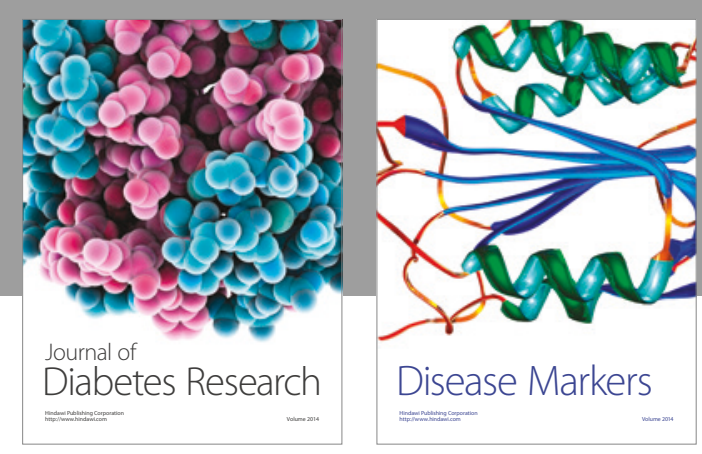

Disease Markers
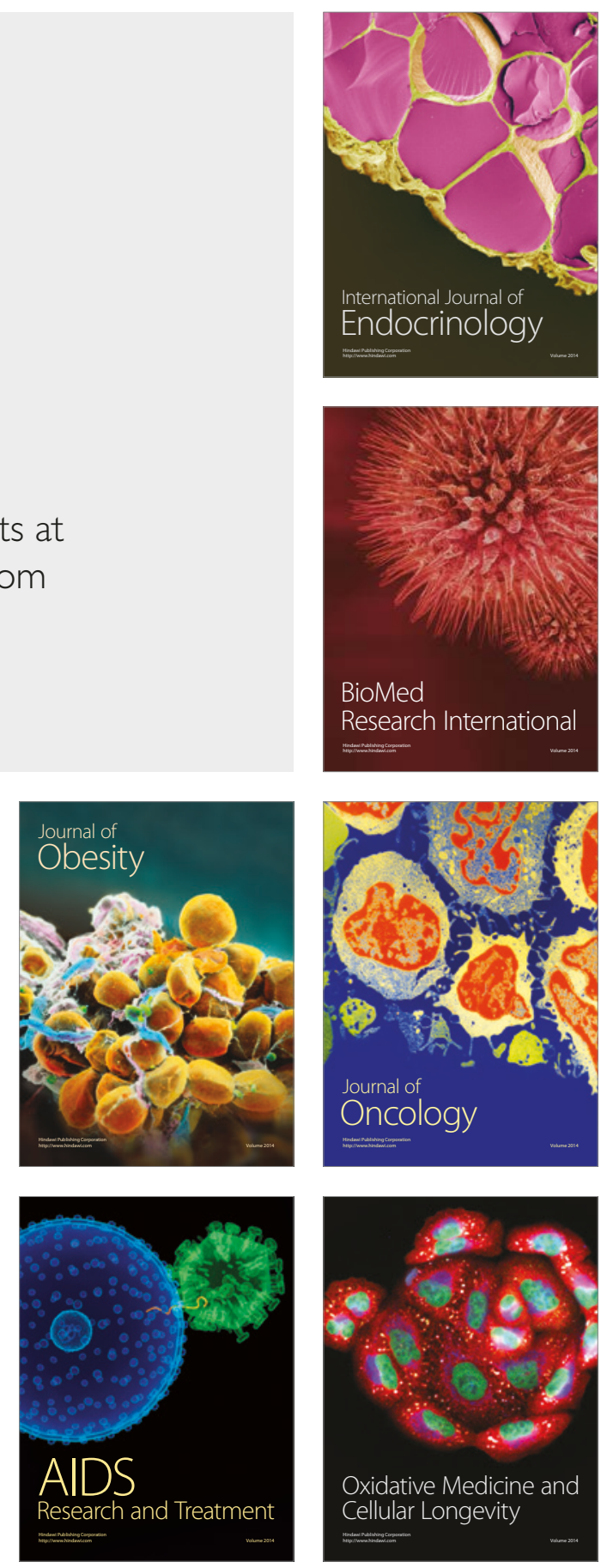